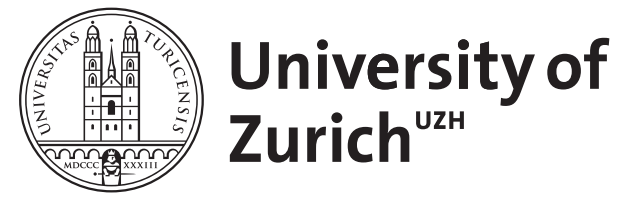

\title{
Möbius rigidity of invariant metrics in boundaries of symmetric spaces of
} rank-1

\author{
Platis, I D ; Schroeder, Viktor
}

\begin{abstract}
Let $\mathbf{H}_{K}^{n}$ denote the symmetric space of rank-1 and of non-compact type and let $d_{\mathfrak{H}}$ be the Korányi metric defined on its boundary. We prove that if $d$ is a metric on $\partial \mathbf{H}_{K}^{n}$ such that all Heisenberg similarities are $d$-Möbius maps, then under a topological condition $d$ is a constant multiple of a power of $d_{\mathfrak{H}}$.
\end{abstract}

DOI: https://doi.org/10.1007/s00605-016-0982-1

Posted at the Zurich Open Repository and Archive, University of Zurich ZORA URL: https://doi.org/10.5167/uzh-128369

Journal Article

Accepted Version

Originally published at:

Platis, I D; Schroeder, Viktor (2017). Möbius rigidity of invariant metrics in boundaries of symmetric spaces of rank-1. Monatshefte fuer Mathematik, 183(2):357-373.

DOI: https://doi.org/10.1007/s00605-016-0982-1 


\title{
MÖBIUS RIGIDITY OF INVARIANT METRICS IN BOUNDARIES OF SYMMETRIC SPACES OF RANK-1
}

\author{
I.D. PLATIS \& V. SCHROEDER
}

\begin{abstract}
Let $\mathbf{H}_{\mathbb{K}}^{n}$ denote the symmetric space of rank-1 and of non-compact type and let $d_{\mathfrak{H}}$ be the Korányi metric defined on its boundary. We prove that if $d$ is a metric on $\partial \mathbf{H}_{\mathbb{K}}^{n}$ such that all Heisenberg similarities are $d$-Möbius maps, then under a topological condition $d$ is a constant multiple of a power of $d_{\mathfrak{H}}$.
\end{abstract}

\section{Introduction and Statement of Results}

Let $(S, d)$ be a metric space and suppose that there exists a remote point $\infty$ such that $\bar{S}=S \cup\{\infty\}$ is compact. We may extend $d$ to the compactification by agreeing that $d(p, \infty)=+\infty$ for every $p \in S$ and also $d(\infty, \infty)=0$. A natural metric cross-ratio $\left|\mathbb{X}^{d}\right|$ is defined for each quadruple of pairwise distinct points $\mathfrak{p}=\left(p_{1}, p_{2}, p_{3}, p_{4}\right)$ by setting

$$
\left|\mathbb{X}^{d}\right|(\mathfrak{p})=\frac{d\left(p_{4}, p_{2}\right)}{d\left(p_{4}, p_{1}\right)} \cdot \frac{d\left(p_{3}, p_{1}\right)}{d\left(p_{3}, p_{2}\right)}
$$

with obvious modifications when one of the points is $\infty$. The Möbius group $\mathcal{M}_{d}=\mathcal{M}_{d}(S)$ is the group of homeomorphisms of $\bar{S}$ which leave $\left|\mathbb{X}^{d}\right|$ invariant for each quadruple $\mathfrak{p}$ of pairwise distinct points of $\bar{S}$. The similarity group (or homothety group) $\operatorname{Sim}_{d}=\operatorname{Sim}_{d}(S)$ is the subgroup of $\mathcal{M}_{d}$ comprising homeomorphisms $\phi$ such that there exists a positive constant $K(\phi)$ which satisfies $d(\phi(p), \phi(q))=K(\phi) \cdot d(p, q)$, for all $p, q \in S$. If $K(\phi)=1$ then $\phi$ is an isometry.

Given two metrics $d_{1}$ and $d_{2}$ on $S$ for which $\infty$ is their remote point, we say that they define the same Möbius structure on $S$ if $\left|\mathbb{X}^{d_{1}}\right|=\left|\mathbb{X}^{d_{2}}\right|$. This happens if and only if $d_{1}$ and $d_{2}$ are homothetic, that is, there exists a $c>0$ such that $d_{1}=c \cdot d_{2}$ (see Lemma 2.1 of [2]). It is immediate that for such metrics we have $\mathcal{M}_{d_{1}}=\mathcal{M}_{d_{2}}$.

In this paper we are concerned with the following problem: Suppose that we are given a representative $d_{1}$ of a Möbius structure on $\bar{S}$ and suppose also that there exists a metric $d_{2}$ defined on $S$ which has the same remote point as $d_{1}$ and also satisfies $\mathcal{M}_{d_{1}} \subseteq \mathcal{M}_{d_{2}}$. Then, assuming that $\mathcal{M}_{d_{1}}$ is sufficiently rich, what can we say about the relation of $d_{1}$ and $d_{2}$ ?

We give a quite precise answer to this question in the case where $\bar{S}$ is the boundary of a symmetric space of rank-1 and of non-compact type and the given Möbius structure is the canonical one, i.e., the one arising from the Korányi metric. We recall below some known facts about the aforementioned notions; for details we refer to Section 2 . Let $\mathbb{K}=\mathbb{R}, \mathbb{C}, \mathbb{H}$ or $\mathbb{O}$ be the set of real, complex, quaternionic and octonionic numbers, respectively. With $\mathbf{H}_{\mathbb{K}}^{n}$ we shall thereafter denote the $n$-dimensional $\mathbb{K}$-hyperbolic space (in the case $\mathbb{K}=\mathbb{O}, n=2$ ). Symmetric spaces of rank-1 and of non-compact type are necessarily the $\mathbb{K}$-hyperbolic spaces. There is a natural conjugation $z \mapsto \bar{z}$ in $\mathbb{K}$ (in the case when $\mathbb{K}=\mathbb{R}$ this is of course the identity map); denote by $\Im(\mathbb{K})$ the subset of $\mathbb{K}$ comprising points $z$ such that $z=-\bar{z}$. The boundary $\partial \mathbf{H}_{\mathbb{K}}^{n}$ is a sphere, isomorphic to the one

Date: 17 March 2016

2010 Mathematics Subject Classifications. 51B10, 51F99.

Key words. Möbius space, invariant metric, boundary of symmetric space. 
point compactification of the $\mathbb{K}$-Heisenberg group $\mathfrak{H}_{\mathbb{K}}$ : That is, the set $\mathbb{K}^{n-1} \times \Im(\mathbb{K})$ endowed with the group multiplication

$$
(\zeta, v) *\left(\zeta^{\prime}, v^{\prime}\right)=\left(\zeta+\zeta^{\prime}, v+v+2 \omega\left(\zeta, \zeta^{\prime}\right)\right)
$$

for each $(\zeta, v),\left(\zeta^{\prime}, v^{\prime}\right) \in \mathfrak{H}_{\mathbb{K}}$. Here, $\omega$ is the usual symplectic form in $\mathbb{K}^{n-1}$. A natural conjugation $J$ is defined on $\mathbb{K}^{n-1} \times \Im(\mathbb{K})$ as the restriction of the conjugation of $\mathbf{H}_{\mathbb{K}}^{n}$; that is, $(\zeta, v) \mapsto(\bar{\zeta},-v)$. There is a gauge $|\cdot|_{\mathbb{K}}$ on $\mathfrak{H}_{\mathbb{K}}$, called the Korányi gauge, which is given for each $(\zeta, v)$ by

$$
|(\zeta, v)|_{\mathbb{K}}=\left|-\|\zeta\|^{2}+v\right|^{1 / 2}=\left(\|\zeta\|^{4}+|v|^{2}\right)^{1 / 4}
$$

The Korányi (or, Korányi-Cygan) metric $d_{\mathfrak{H}}$ is then defined in $\mathfrak{H}_{\mathbb{K}}$ by

$$
d_{\mathfrak{H}}\left((\zeta, v),\left(\zeta^{\prime}, v^{\prime}\right)\right)=\left|\left(\zeta^{\prime}, v^{\prime}\right)^{-1} *(\zeta, v)\right|_{\mathbb{K}},
$$

for each $(\zeta, v),\left(\zeta^{\prime}, v^{\prime}\right) \in \mathfrak{H}_{\mathbb{K}}$. The canonical Möbius structure of $\partial \mathbf{H}_{\mathbb{K}}^{n}$ is the one arising from $d_{\mathfrak{H}}$. The Möbius group $\mathcal{M}_{d_{\mathfrak{H}}}$ of the metric $d_{\mathfrak{H}}$ is generated by the similarities of $d_{\mathfrak{H}}$ and an inversion $I$ defined on $\partial \mathbf{H}_{\mathbb{K}}^{n}$, see Section 2 for details.

One of our main results can be stated in the following way:

Theorem. Let $d$ be a metric on $\partial \mathbf{H}_{\mathbb{K}}^{n}$, which induces the same topology and has the same remote point as $d_{\mathfrak{H}}$. Assume further that $\mathcal{M}_{d}=\mathcal{M}_{d_{\mathfrak{H}}}$. Then there exist $\alpha \in(0,1]$ and $\beta>0$ such that $d=\beta \cdot d_{\mathfrak{H}}^{\alpha}$.

This special result is embedded in much more general statements: Suppose that we are given a metric $d$ in $\partial \mathbf{H}_{\mathbb{K}}^{n}$ such that:

$(\operatorname{Sim}) \operatorname{Sim}_{d_{\mathfrak{H}}} \subseteq \operatorname{Sim}_{d}$

We shall further presuppose the next quite plausible topological condition:

(Top) If a sequence of points $p_{\nu} \in \partial \mathbf{H}_{\mathbb{K}}^{n}, \nu \in \mathbb{N}$, is converging in the $d_{\mathfrak{H}}$-topology of $\partial \mathbf{H}_{\mathbb{K}}^{n}$, then it converges in the $d$-topology of $\partial \mathbf{H}_{\mathbb{K}}^{n}$.

Before stating our results, we list the following set of conditions:

(Inv) Inversion $I$ is in $\mathcal{M}_{d}$.

( $\alpha$-Höl) There exists a constant $\alpha \in(0,1]$ such that the identity map of $\left(\partial \mathbf{H}_{\mathbb{K}}^{n}, d_{\mathfrak{H}}\right) \rightarrow\left(\partial \mathbf{H}_{\mathbb{K}}^{n}, d\right)$ is Hölder continuous with exponent $\alpha$. Explicitly, there exists a $\beta>0$ such that for each $p, q \in \partial \mathbf{H}_{\mathbb{K}}^{n}$,

$$
d(p, q) \leq \beta \cdot d_{\mathfrak{H}}^{\alpha}(p, q) .
$$

(G) There exists an $\alpha \in(0,1]$ such that for each $p=(\zeta, v) \in \mathfrak{H}_{\mathbb{K}}, \mathbb{K} \neq \mathbb{R}$,

$$
d^{4 / \alpha}(o, p)=d^{4 / \alpha}\left(o, \Pi_{\mathbb{K}^{n-1}}(p)\right)+d^{4 / \alpha}\left(\Pi_{\mathbb{K}^{n-1}}(p), \Pi_{\Im(\mathbb{K})}(p)\right) .
$$

Here, $o=\left(\mathbf{0}_{\mathbb{K}^{n-1}}, \mathbf{0}_{\Im(\mathbb{K})}\right)$ is the origin of $\mathfrak{H}_{\mathbb{K}}$, where $\mathbf{0}_{\mathbb{K}^{n-1}}$ and $\mathbf{0}_{\Im(\mathbb{K})}$ are the origins of $\mathbb{K}^{n-1}$ and $\Im(\mathbb{K})$, respectively. Moreover, $\Pi_{\mathbb{K}^{n-1}}, \Pi_{\Im(\mathbb{K})}$ are defined respectively by $\Pi_{\mathbb{K}^{n-1}}(p)=\zeta$ and $\Pi_{\Im(\mathbb{K})}(p)=v$.

(Eq) The metric $d$ satisfies

$$
d(o,(1,0))=d(o,(0,1)) .
$$

Here, $(1,0)=\left(\mathbf{e}_{1}, \mathbf{0}_{\Im(\mathbb{K})}\right)$, where $\mathbf{e}_{1}=(1,0, \ldots, 0)$ is the first coordinate vector of $\mathbb{K}^{n-1}$ and $(0,1)=\left(\mathbf{0}_{\mathbb{K}^{n-1}}, \mathbf{f}_{1}\right)$ where $\mathbf{f}_{1}$ is the first coordinate vector of $\Im(\mathbb{K})$.

(biLip) The metric $d$ is bi-Lipschitz equivalent to $d_{\mathfrak{H}}^{\alpha}$ for some $\alpha \in(0,1]$.

$\left(\alpha\right.$-Met) There exist constants $\beta>0$ and $\alpha \in(0,1]$ such that $d=\beta \cdot d_{\mathfrak{H}}^{\alpha}$. 
Our first theorem assumes the least number of conditions for $d$, that is, (Sim) and (Top).

Theorem 1.1. Let $d$ be a metric in $\mathfrak{H}_{\mathbb{K}}$ and denote again by dits extension to $\partial \mathbf{H}_{\mathbb{K}}^{n}=\mathfrak{H} \cup\{\infty\}$. Then the following hold:

(1) If $\mathbb{K}=\mathbb{R}$, conditions (Sim) and (Top) together imply ( $\alpha$-Met).

(2) If $\mathbb{K} \neq \mathbb{R}$, conditions (Sim) and (Top) together imply ( $\alpha$-Höl).

We note the significant difference between the real case and all the other cases. With the least possible assumptions, the metric $d$ is a power of the Euclidean metric when $\mathbb{K}=\mathbb{R}$. The picture is entirely different in case when $\mathbb{K} \neq \mathbb{R}$; metrics which satisfy ( $\alpha$-Höl) may be of nature entirely different from the one of a power of $d_{\mathfrak{H}}$ (e.g., the Carnot-Carathéodory metric). It is therefore quite necessary to add more assumptions for $d$ in this case. To that end, the strongest version of our first main result for the case $\mathbb{K} \neq \mathbb{R}$ follows:

Theorem 1.2. With the assumptions of Theorem 1.1, suppose $\mathbb{K} \neq \mathbb{R}$. Then conditions (Sim), (Top) and (Inv) together imply ( $\alpha$-Met).

But again, adding (Inv) to our basic assumptions seems to be excessive for the proof of ( $\alpha$ Met); in the case $\mathbb{K}=\mathbb{R}$ (Inv) holds a posteriori. It is natural therefore to ask if (Inv) is really necessary. Theorem 1.1 tells us that we obtain a rather weak result if we entirely drop (Inv). It turns out though that ( $\alpha$-Met) follows by replacing (Inv) with $(\mathbf{G})$ and $(\mathbf{E q})$. In fact, we have:

Theorem 1.3. With the assumptions of Theorem 1.1, suppose $\mathbb{K} \neq \mathbb{R}$. Then:

(1) Conditions (Sim), (Top) and (G) together imply (biLip).

(2) Conditions (Sim), (Top), (G) and (Eq) together imply ( $\alpha$-Met).

Therefore, (Inv) follows as a side result of Theorem 1.3 and we may further observe that $(\mathbf{G})$ and (Eq) hold vacuously in the real case. Moreover, (G) can be replaced with an equivalent statement which is the closest to parallelogram law in the $\mathbb{K}$-Heisenberg group setting, $\mathbb{K} \neq \mathbb{R}$. For this set for each $p \in \mathfrak{H}$,

$$
|p|=d(o, p) \text {. }
$$

It turns out that if ( $\mathbf{S i m}$ ) holds, then $(\mathbf{G})$ is equivalent to the following condition, see Proposition 3.4:

(P-L) For $\alpha \in(0,1]$ and for each $p, q \in \mathfrak{H}$,

$$
\begin{aligned}
|p * q|^{4 / \alpha}+\left|p^{-1} * q\right|^{4 / \alpha}+\left|p * q^{-1}\right|^{4 / \alpha}+\left|p^{-1} * q^{-1}\right|^{4 / \alpha} & = \\
2\left(\left|\Pi_{\mathbb{K}^{n-1}}(p * q)\right|^{4 / \alpha}+\left|\Pi_{\mathbb{K}^{n-1}}\left(p^{-1} * q\right)\right|^{4 / \alpha}\right) & + \\
\left|\Pi_{\Im(\mathbb{K})}(p * q)\right|^{4 / \alpha}+\left|\Pi_{\Im(\mathbb{K})}\left(p^{-1} * q\right)\right|^{4 / \alpha}+\left|\Pi_{\Im(\mathbb{K})}\left(p * q^{-1}\right)\right|^{4 / \alpha}+\left|\Pi_{\Im(\mathbb{K})}\left(p^{-1} * q^{-1}\right)\right|^{4 / \alpha}, &
\end{aligned}
$$

where $\Pi_{\mathbb{K}^{n-1}}$ and $\Pi_{\Im(\mathbb{K})}$ are the projections of $\mathfrak{H}$ to $\mathbb{K}^{n-1}$ and $\Im(\mathbb{K})$, respectively.

Thus an equivalent to the second statement of Theorem $1.3(2)$ is:

$\left(2^{\prime}\right)$ Conditions (Sim), (Top), (P-L) and (Eq) together imply ( $\alpha$-Met).

Recall now that a metric $d$ defined in a space $S$ is Ptolemaean if for each quadruple of points $\mathfrak{p}=(p, q, r, s)$ of $S$ the following inequality is satisfied for all possible permutations of points in $\mathfrak{p}$ :

$$
d(p, r) \cdot d(q, s) \leq d(p, q) \cdot d(r, s)+d(p, s) \cdot d(r, q) .
$$

A Ptolemaean circle is a subset $\sigma$ of $S$ which is topologically equivalent to the unit circle $S^{1}$ and for each quadruple of points $\mathfrak{p}=(p, q, r, s)$ of $\sigma$ such that $p$ and $r$ separate $q$ and $s$, Inequality 1.2 
holds as an equality. It is well known that $\left(\partial \mathbf{H}_{\mathbb{K}}^{n}, d_{\mathfrak{H}}\right)$ is Ptolemaean, see for instance [12]; therefore it is natural to ask which of the metrics $d$ who satisfy ( $\alpha$-Met) satisfies also:

(Ptol) The metric $d$ is Ptolemaean.

It turns out that we will also need:

(Circ) The metric $d$ has a Ptolemaean circle.

We have the following corollary to Theorem 1.3:

Theorem 1.4. Condition ( $\alpha$-Met) implies (Ptol) and (Ptol) together with (Circ) are equivalent to (1-Met). Therefore a metric d in $\partial \mathbf{H}_{\mathbb{K}}^{n}$ which satisfies conditions:

(1) (Sim), (Top) and (Circ) if $\mathbb{K}=\mathbb{R}$ and

(2) (Sim), (Top), (P-L) or (G), (Eq) and (Circ) if $\mathbb{K} \neq \mathbb{R}$,

is necessarily a constant multiple of the Korányi metric $d_{\mathfrak{H}}$.

This is in the spirit of the old result of Schoenberg, see [14]. That particular result was on metrics which were derived from semi-norms which also share Ptolemaean property.

Part of this work was carried out while IDP was visiting University of Zürich, Switzerland. Hospitality of Institüt für Mathematik, University of Zürich, is gratefully appreciated.

\section{Preliminaries}

This section is divided in two parts. In the first part (Section 2.1), we state for clarity some known results about the Korányi metric in $\partial \mathbf{H}_{\mathbb{K}}^{n}$ and its properties. In the second part (Section 2.2), we state in brief some elementary facts concerning Möbius geometry, mainly focusing on properties of the similarity group.

2.1. The $\mathbb{K}$-Heisenberg group $\mathfrak{H}_{\mathbb{K}}$ and the Korányi metric $d_{\mathfrak{H}}$. The following are well known; we refer the reader to the classical book of Mostow, [10], or to [12]. Another useful reference for the case $\mathbb{K}=\mathbb{C}$ is the book of Goldman, [7]. For the octonionic case in particular, we refer to [1] and to [11].

We shall use hereafter the following notation $(n>1)$ :

$$
\mathrm{G}_{\mathbb{K}}=\left\{\begin{array}{lr}
\mathrm{SO}(n, 1) & \text { if } \mathbb{K}=\mathbb{R}, \\
\mathrm{SU}(n, 1) & \text { if } \mathbb{K}=\mathbb{C}, \\
\mathrm{Sp}(n, 1) & \text { if } \mathbb{K}=\mathbb{H}, \\
F_{4(-20)} & \text { if } \mathbb{K}=\mathbb{O}(n=2) .
\end{array}\right.
$$

Also,

$$
\mathrm{F}(n)=\left\{\begin{array}{cc}
\mathrm{SO}(n) & \text { if } \mathbb{K}=\mathbb{R}, \\
\mathrm{SU}(n) & \text { if } \mathbb{K}=\mathbb{C}, \\
\operatorname{Sp}(n) & \text { if } \mathbb{K}=\mathbb{H}, \\
\operatorname{Spin}_{7}(\mathbb{R}) & \text { if } \mathbb{K}=\mathbb{O}(n=2) .
\end{array}\right.
$$

$\mathbb{K}$-hyperbolic space $\mathbf{H}_{\mathbb{K}}^{n}$ is $\mathrm{G}_{\mathbb{K}} / \mathrm{F}(n)$. The metric $d_{\mathfrak{H}}$ as given in Equation (1.1) is invariant under the following transformations (and their extensions to $\infty$ ).

(1) Left translations which come from the action of $\mathfrak{H}_{\mathbb{K}}$ on itself: For any fixed point $\left(\zeta^{\prime}, v^{\prime}\right) \in \mathfrak{H}_{\mathbb{K}}$ let

$$
T_{\left(\zeta^{\prime}, v^{\prime}\right)}(\zeta, v)=\left(\zeta^{\prime}, v^{\prime}\right) *(\zeta, v), \quad T_{\left(\zeta^{\prime}, v^{\prime}\right)}(\infty)=\infty .
$$

In the particular case where $\mathbb{K}=\mathbb{R}, d_{\mathfrak{H}}$ is also invariant under the right translations. 
(2) Rotations: For the cases $\mathbb{K} \neq \mathbb{O}$, these come from the action of $\mathrm{F}(n-1)$ on $\mathbb{K}^{n-1}$. Specifically, given a $U \in \mathrm{F}(n-1), \mathbb{K} \neq \mathbb{O}$, we define

$$
S_{U}(\zeta, v)=(U \cdot \zeta, v), \quad S_{U}(\infty)=\infty .
$$

Only in the case where $\mathbb{K}=\mathbb{H}$ we have the action of $\mathrm{F}(1)=\mathrm{Sp}(1)$ given by

$$
\left(\zeta_{1}, \ldots, \zeta_{n-1}, v\right) \mapsto\left(\mu \zeta_{1} \mu^{-1}, \ldots, \mu \zeta_{n-1} \mu^{-1}, \mu v \mu^{-1}\right), \quad \mu \in \operatorname{Sp}(1) ;
$$

observe that in all other cases this action is vacuous. In the particular case $\mathbb{K}=\mathbb{O}$, for given unit imaginary octonion $\mu$, let

$$
S_{\mu}(\zeta, v)=(\zeta \cdot \bar{\mu}, \mu v \bar{\mu}), \quad S_{\mu}(\infty)=\infty .
$$

We stress at this point that in general $S_{\mu} \circ S_{\nu} \neq S_{\mu \nu}$ for $\mu, \nu$ unit imaginary octonions. The group generated by transformations $S_{\mu}$ is the compact group $\operatorname{Spin}_{7}(\mathbb{R})$.

All these actions generate the group $\operatorname{Isom}_{d_{\mathfrak{H}}}\left(\partial\left(\mathbf{H}_{\mathbb{K}}^{n}\right)\right.$ of (orientation-preserving) $d_{\mathfrak{H}}$-isometries; this acts transitively on $\mathfrak{H}_{\mathbb{K}}$. We also consider two other kinds of transformations of $\partial \mathbf{H}_{\mathbb{K}}^{n}$.

(3) Dilations: If $\delta \in \mathbb{R}_{*}^{+}$we define

$$
D_{\delta}(\zeta, v)=\left(\delta \zeta, \delta^{2} v\right), \quad D_{\delta}(\infty)=\infty .
$$

(In the octonionic case, $\delta^{2}$ is used in [11] instead of $\delta$, but the model for $\mathfrak{H}_{\mathbb{O}}$ is somewhat different). One verifies that for every $(\zeta, v),\left(\zeta^{\prime}, v^{\prime}\right) \in \partial \mathbf{H}_{\mathbb{K}}^{n}$ we have

$$
d_{\mathfrak{H}}\left(D_{\delta}(\zeta, v), D_{\delta}\left(\zeta^{\prime}, v^{\prime}\right)\right)=\delta \cdot d_{\mathfrak{H}}\left((\zeta, v),\left(\zeta^{\prime}, v^{\prime}\right)\right) .
$$

Thus the metric $d_{\mathfrak{H}}$ is scaled up to multiplicative constants by the action of dilations. We mention here that together with $d_{\mathfrak{H}}$-isometries, dilations generate the $d_{\mathfrak{H}}$-similarity group $\operatorname{Sim}_{d_{\mathfrak{H}}}\left(\partial \mathbf{H}_{\mathbb{K}}^{n}\right)$.

(4) Inversion $I$ is given by

$$
I(\zeta, v)=\left(\zeta\left(-\|\zeta\|^{2}+v\right)^{-1}, \bar{v}\left|-\|\zeta\|^{2}+v\right|^{-2}\right), \text { if }(\zeta, v) \neq o, \infty, \quad I(o)=\infty, I(\infty)=o .
$$

Inversion $I$ is an involution of $\partial \mathbf{H}_{\mathbb{K}}^{n}$. Moreover, for all $p=(\zeta, v), p^{\prime}=\left(\zeta^{\prime}, v^{\prime}\right) \in \mathfrak{H}_{\mathbb{K}} \backslash\{o\}$ we have

$$
d_{\mathfrak{H}}(I(p), o)=\frac{1}{d_{\mathfrak{H}}(p, o)}, \quad d_{\mathfrak{H}}\left(I(p), I\left(p^{\prime}\right)\right)=\frac{d_{\mathfrak{H}}\left(p, p^{\prime}\right)}{d_{\mathfrak{H}}(p, o) d_{\mathfrak{H}}\left(o, p^{\prime}\right)}
$$

The similarity group $\operatorname{Sim}_{d_{\mathfrak{H}}}\left(\partial \mathbf{H}_{\mathbb{K}}^{n}\right)$ is the semidirect product $\mathbb{R} \rtimes \operatorname{Isom}_{d_{\mathfrak{H}}}\left(\partial \mathbf{H}_{\mathbb{K}}^{n}\right)$. The group generated by $\operatorname{Sim}_{d_{\mathfrak{H}}}\left(\partial \mathbf{H}_{\mathbb{K}}^{n}\right)$ and inversion $I$ is isomorphic to $G_{\mathbb{K}}$ (with the exception of the case $\mathbb{K}=\mathbb{R}, n$ odd: in this case, $I$ reverses orientation). Given two distinct points on the boundary, we can find an element of $G_{\mathbb{K}}$ mapping those points to $o$ and $\infty$ respectively; in particular $G_{\mathbb{K}}$ acts doubly transitively on the boundary. In the exceptional case where $\mathbb{K}=\mathbb{R}$, the action of $G_{\mathbb{R}}$ is triply transitive; this follows from the fact that we can map three distinct points of the boundary to the points $o, \infty$ and $\mathbf{e}_{1}$, respectively.

2.2. Möbius Group, Similarity Group. Recall from the introduction that in the most general setting we start from a mere metric space $(S, d)$ and we suppose that there is a remote point $\infty$ such that:

(1) $\bar{S}=S \cup\{\infty\}$ is compact;

(2) $d$ is extended to the compactification $\bar{S}$ by setting $d(p, \infty)=+\infty$, for each $p \in S$ and also $d(\infty, \infty)=0$. 
The notion of metric cross-ratio is next. The following proposition may be verified straightforwardly, detailed discussions about cross-ratios may be found among others in [5], [6], [9] and [12]:

Proposition 2.1. Let $(S, d)$ be a metric space with a remote point $\infty$. Denote by $\mathcal{C}$ the space $S \times S \times S \times S \backslash\{$ diagonals $\}$. Then the map $\left|\mathbb{X}^{d}\right|: \mathcal{C} \rightarrow \mathbb{R}_{*}^{+}$defined for each $\mathfrak{p}=\left(p_{1}, p_{2}, p_{3}, p_{4}\right) \in \mathcal{C}$ by

$$
\left|\mathbb{X}^{d}\right|(\mathfrak{p})=\frac{d\left(p_{4}, p_{2}\right)}{d\left(p_{4}, p_{1}\right)} \cdot \frac{d\left(p_{3}, p_{1}\right)}{d\left(p_{3}, p_{2}\right)}
$$

satisfies the following:

(1) Symmetries:

$$
\left|\mathbb{X}^{d}\right|\left(p_{1}, p_{2}, p_{3}, p_{4}\right)=\left|\mathbb{X}^{d}\right|\left(p_{2}, p_{1}, p_{4}, p_{3}\right)=\left|\mathbb{X}^{d}\right|\left(p_{3}, p_{4}, p_{1}, p_{2}\right)=\left|\mathbb{X}^{d}\right|\left(p_{4}, p_{3}, p_{2}, p_{1}\right) .
$$

(2) Let

$$
\left|\mathbb{X}_{1}^{d}\right|(\mathfrak{p})=\left|\mathbb{X}^{d}\right|\left(p_{1}, p_{2}, p_{3}, p_{4}\right), \quad \text { and } \quad\left|\mathbb{X}_{2}^{d}\right|(\mathfrak{p})=\left|\mathbb{X}^{d}\right|\left(p_{1}, p_{3}, p_{2}, p_{4}\right)
$$

Then

$$
\begin{aligned}
& \left|\mathbb{X}^{d}\right|\left(p_{1}, p_{3}, p_{4}, p_{2}\right)=\frac{1}{\left|\mathbb{X}_{2}^{d}\right|}, \quad\left|\mathbb{X}^{d}\right|\left(p_{1}, p_{4}, p_{3}, p_{2}\right)=\frac{\left|\mathbb{X}_{1}^{d}\right|}{\left|\mathbb{X}_{2}^{d}\right|} \\
& \left|\mathbb{X}^{d}\right|\left(p_{1}, p_{2}, p_{4}, p_{3}\right)=\frac{1}{\left|\mathbb{X}_{1}^{d}\right|}, \quad\left|\mathbb{X}^{d}\right|\left(p_{1}, p_{4}, p_{2}, p_{3}\right)=\frac{\left|\mathbb{X}_{2}^{d}\right|}{\left|\mathbb{X}_{1}^{d}\right|}
\end{aligned}
$$

Thus for any possible permutation $\tilde{\mathfrak{p}}$ of points of a given quadruple $\mathfrak{p},\left|\mathbb{X}^{d}(\tilde{\mathfrak{p}})\right|$ depends only on $\left|\mathbb{X}_{1}^{d}\right|(\mathfrak{p})$ and $\left|\mathbb{X}_{1}^{d}\right|(\mathfrak{p})$.

A homeomorphism $\phi: S \rightarrow S$ shall be called a $d$-Möbius map if $\left|\mathbb{X}^{d}(\mathfrak{p})\right|=\left|\mathbb{X}^{d}(\phi(\mathfrak{p}))\right|$ for each $\mathfrak{p}=\left(p_{1}, p_{2}, p_{3}, p_{4}\right) \in \mathcal{C}$, where, by $\phi(\mathfrak{p})$ we denote the quadruple $\left(\phi\left(p_{1}\right), \phi\left(p_{2}\right), \phi\left(p_{3}\right), \phi\left(p_{4}\right)\right)$. This is equivalent to say that for each given quadruple $\mathfrak{p}$,

$$
\left|\mathbb{X}_{1}^{d}\right|(\phi(\mathfrak{p}))=\left|\mathbb{X}_{1}^{d}\right|(\mathfrak{p}) \quad \text { and } \quad\left|\mathbb{X}_{2}^{d}\right|(\phi(\mathfrak{p}))=\left|\mathbb{X}_{2}^{d}\right|(\mathfrak{p}) .
$$

The set of all Möbius maps of $S$ form a group $\mathcal{M}_{d}=\mathcal{M}_{d}(S)$, which we shall call the Möbius group of $(S, d)$. In particular, we consider the subset $\operatorname{Sim}_{d}$ of $\mathcal{M}_{d}$ consisting of similarities: An element $\phi \in \mathcal{M}_{d}$ is a similarity, if there exists a $K_{\phi}>0$ such that for every $p, q \in S$

$$
d(\phi(p), \phi(q))=K_{\phi} \cdot d(p, q) .
$$

By definition, $K_{\phi}=K(\phi)$ is independent of the choice of points.

Proposition 2.2. The set $\operatorname{Sim}_{d}$ is a subgroup of $\mathcal{M}_{d}$. There exists a group homomorphism $K: \operatorname{Sim}_{d} \rightarrow \mathbb{R}_{*}^{+}$given for each $\phi \in \operatorname{Sim}_{d}$ by

$$
K(\phi)=K_{\phi}
$$

Here $\mathbb{R}_{*}^{+}$is the multiplicative topological group of the positive reals, inheriting its topology from the real line.

Proof. We show that $\operatorname{Sim}_{d}$ is a subgroup of $\mathcal{M}_{d}$. If $\phi, \psi \in \operatorname{Sim}_{d}$, then for every $p, q \in S$,

$$
d(\phi(p), \phi(q))=K_{\phi} \cdot d(p, q) \text { and } d(\psi(p), \psi(q))=K_{\psi} \cdot d(p, q) .
$$


Therefore for each $p, q \in S$,

$$
\begin{aligned}
d((\phi \circ \psi)(p),(\phi \circ \psi)(q)) & =d((\phi(\psi(p)),(\phi(\psi(q))) \\
& =K_{\phi} \cdot d(\psi(p), \psi(q)) \\
& =K_{\phi} \cdot K_{\psi} \cdot d(p, q) .
\end{aligned}
$$

Since this shows that $\phi \circ \psi$ is a similarity, we must also have

$$
d((\phi \circ \psi)(p),(\phi \circ \psi)(q))=K_{\phi \circ \psi} \cdot d(p, q) .
$$

Hence we also must have $K_{\phi^{-1}}=\left(K_{\phi}\right)^{-1}$.

We call $K$ the similarity homomorphism. Under our assumptions for $(S, d)$ in the beginning of this section, we endow $\mathcal{M}_{d}$ with the compact-open topology and accordingly $\operatorname{Sim}_{d}$ inherits the relative topology. We then have:

Proposition 2.3. The similarity homomorphism $K$ is continuous.

Proof. For any $\phi \in \operatorname{Sim}_{d}$ we have

$$
K(\phi)=\frac{d(\phi(p), \phi(q))}{d(p, q)}
$$

for any fixed $p, q \in S$. Now the map $\operatorname{Sim}_{d} \rightarrow S, \phi \mapsto \phi(p)$, is continuous in the compact-open topology for any fixed $p$, and $d: S \times S \rightarrow \mathbb{R}^{+}$is continuous by definition.

We are interested in the stabiliser of $\infty$ in $\mathcal{M}_{d}$, that is,

$$
\operatorname{Stab}_{d}(\infty):=\operatorname{Stab}_{\mathcal{M}_{d}}(\infty)=\left\{\phi \in \mathcal{M}_{d} \mid \phi(\infty)=\infty\right\}
$$

Proposition 2.4. $\operatorname{Stab}_{d}(\infty)=\operatorname{Sim}_{d}$.

Proof. We show first that $\operatorname{Sim}_{d} \subseteq \operatorname{Stab}_{d}(\infty)$. Supposing the contrary, assume that there exists a $\phi \in \operatorname{Sim}_{d}$ such that $\phi(\infty)=p \in S$. Then for any $q \in S, q \neq p$, we would have

$$
+\infty=d\left(\phi^{-1}(p), \phi^{-1}(q)\right)=K_{\phi^{-1}} \cdot d(p, q),
$$

a contradiction.

To show that $\operatorname{Stab}_{d}(\infty) \subseteq \operatorname{Sim}_{d}$ we only have to prove that any element $\phi \in \operatorname{Stab}_{d}(\infty)$ is a similarity. For this, we consider an arbitrary $\phi \in \operatorname{Stab}_{d}(\infty)$ and we fix two arbitrary points $p, q \in S$. We also consider the quantity

$$
K(\phi, p, q)=\frac{d(\phi(p), \phi(q))}{d(p, q)} .
$$

Now for any $r \in S$, let $\mathfrak{p}=(r, q, \infty, p)$. Relations

$$
\left|\mathbb{X}_{1}^{d}\right|(\mathfrak{p})=\left|\mathbb{X}_{1}^{d}\right|(\phi(\mathfrak{p})) \text { and }\left|\mathbb{X}_{2}^{d}\right|(\mathfrak{p})=\left|\mathbb{X}_{2}^{d}\right|(\phi(\mathfrak{p}))
$$

yield

$$
K(\phi, p, q)=K(\phi, p, r) \text { and } K(\phi, p, q)=K(\phi, q, r) .
$$

From the left equality it follows that $K(\phi, p, q)$ does not depend on $q$. But then the right equality shows that it also does not depend on $p$. Since $p, q$ are arbitrary, $K=K(\phi)$ and the proof is complete.

In view of Propositions 2.2 and 2.4 we obtain: 
Corollary 2.5. If the similarity homomorphism $K$ is onto, then

$$
\operatorname{Sim}_{d} / \operatorname{Isom}_{d} \simeq \mathbb{R}_{*}^{+},
$$

where $\mathrm{Isom}_{d}$ is the subgroup of $\mathcal{M}_{d}$ consisting of isometries and $\mathbb{R}_{*}^{+}$is the multiplicative group of positive real numbers. Thus we have the short exact sequence

$$
1 \rightarrow \operatorname{Isom}_{d} \rightarrow \operatorname{Sim}_{d} \rightarrow \mathbb{R}_{*}^{+} \rightarrow 1 .
$$

Remark 2.6. Note that if there exists an inverse $L: \mathbb{R}_{*}^{+} \rightarrow \operatorname{Sim}_{d}$ to the similarity homomorphism $K$ of the short exact sequence 2.2 such that $L \circ K$ is the identity homomorphism when restricted to $\mathrm{Isom}_{d}$, then by Splitting Lemma, see [8], we have

$$
\operatorname{Sim}_{d} \simeq \mathbb{R}_{*}^{+} \rtimes \operatorname{Isom}_{d}
$$

the semidirect product of $R_{*}^{+}$and Isom $_{d}$. This is for instance the case where $S=\partial \mathbf{H}_{\mathbb{K}}^{n}$ and $d=d_{\mathfrak{H}}$ : $L$ maps each positive $\delta$ to $D_{\delta}$ and $L \circ K=i d$. when restricted to $\operatorname{Isom}_{d_{\mathfrak{H}}}$.

We close this section with some remarks concerning inversions. In general, an inversion between two distinct points $r, r^{\prime} \in \bar{S}$ is a map $\phi_{r, r^{\prime}} \in \mathcal{M}_{d}$ such that $\phi^{2}=i d$. and $\phi(r)=r^{\prime}$. Of course, an inversion may or may not exist in an arbitrary $\mathcal{M}_{d}$. But if it does, it satisfies properties listed in the next proposition; for simplicity, we only treat the case where $r=\infty$ and $r^{\prime}=o$ is an arbitrary point in $S$.

Proposition 2.7. Let $\phi \in \mathcal{M}_{d}$ be an inversion between o and $\infty$. Then there exists a $\beta>0$ such that

$$
d(o, p) \cdot d(o, \phi(p))=\beta^{2},
$$

for each $p \in S$ other than $o, \infty$. Moreover,

$$
d(\phi(p), \phi(q))=\frac{d(p, q)}{d(o, p) \cdot d(o, q)} \cdot \beta^{2},
$$

for each $p, q \in S$ other than $o, \infty$.

Proof. We consider two arbitrary points $p, q \in S$ other than $o$ and $\infty$ and the quadruple $\mathfrak{p}=(\infty, o$, $p, q)$. Since $\phi \in \mathcal{M}_{d}$ we have $\left|\mathbb{X}_{1}^{d}\right|(\mathfrak{p})=\left|\mathbb{X}_{1}^{d}\right|(\phi(\mathfrak{p}))$, which yields

$$
\frac{d(o, q)}{d(o, p)}=\frac{d(\phi(p), o)}{d(\phi(q), o)} \text {. }
$$

Thus the quantity $d(o, p) \cdot d(0, \phi(p))$ is constant for each $p$. By setting $p=p_{0}$ for some arbitrary $p_{0}$ other that $o$ and $\infty$ and by letting $\beta$ be the positive square root of $d\left(o, p_{0}\right) \cdot d\left(0, \phi\left(p_{0}\right)\right)$, we obtain the first relation of our proposition.

On the other hand, from $\left|\mathbb{X}_{2}^{d}\right|(\mathfrak{p})=\left|\mathbb{X}_{2}^{d}\right|(\phi(\mathfrak{p}))$, we have

$$
\frac{d(p, q)}{d(o, p)}=\frac{d(\phi(p), \phi(q))}{d(o, \phi(q))} .
$$

Therefore,

$$
d(\phi(p), \phi(q))=d(o, \phi(q)) \cdot \frac{d(p, q)}{d(o, p)}=\frac{d(p, q)}{d(o, p) \cdot d(o, q)} \cdot \beta^{2} .
$$

Note finally that from Proposition 2.7 it follows that inversion $\phi$ leaves invariant the metric sphere

$$
S_{d}^{\beta}=\{p \in S \mid d(p, o)=\beta\}
$$




\section{MöBius Rigidity}

In this section we prove our results. In Section 3.1 we prove an elementary lemma which will lead us to the proof of Theorems 1.1, 1.2, 1.3 and 1.4 which are in Sections 3.2, 3.3, 3.4 and 3.5, respectively.

3.1. The Preparatory Lemma. The following holds.

Lemma 3.1. We refer to the conditions stated in the introduction. Then conditions (Sim) and (Top) together imply the following.

(1) If $D_{\delta}$ is a dilation, then $K(\delta)=\delta^{\alpha}$, where $\alpha \in(0,1]$.

(2) The rotational group $\mathrm{F}(n-1)$ is in $\operatorname{Isom}_{d}\left(\partial \mathbf{H}_{\mathbb{K}}^{n}\right)$.

(3) The group of left translations of $\mathfrak{H}_{\mathbb{K}}$ is in $\operatorname{Isom}_{d}\left(\partial \mathbf{H}_{\mathbb{K}}^{n}\right)$.

Proof. To prove (1), we will show that if $D_{d}$ is a dilation, then there exists an $\alpha \in(0,1]$ such that for each $p, q$,

$$
d\left(D_{\delta}(p), D_{\delta}(q)\right)=\delta^{\alpha} \cdot d(p, q) .
$$

For this, set $L=\log K$. Then $L$ is a continuous function satisfying the functional equation

$$
L\left(\delta_{1} \cdot \delta_{2}\right)=L\left(\delta_{1}\right)+L\left(\delta_{2}\right), \quad \text { for all } \delta_{1}, \delta_{2}>0 .
$$

Hence $L(\delta)=\alpha \log (\delta)$ and therefore $K(\delta)=\delta^{\alpha}$ for some non-zero $\alpha$ as desired. Now $\alpha$ cannot be negative, for if that was the case then for every $\delta>0$ we would have $d(o,(\delta, 0))=\delta^{\alpha} \cdot d(o,(1,0))$, where we have written $\left(\mathbf{e}_{1}, \mathbf{0}_{\Im(\mathbb{K})}\right)=(1,0),\left(\delta \mathbf{e}_{1}, \mathbf{0}_{\Im(\mathbb{K})}\right)=(\delta, 0)$. Since the function $f(\delta)=$ $d(o,(\delta, 0))$ is continuous due to the continuity of $d$, by letting $\delta$ tending to $+\infty$ we have a contradiction. The proof that $\alpha$ is in $(0,1]$ lies after the proof of $(3)$.

To prove (2), we observe that from condition (Top) we have that the group $\mathrm{F}(n-1)$ is compact in the compact-open topology arising from $d$. Therefore its $K$-image in the multiplicative group $\mathbb{R}_{*}^{+}$has to be compact, i.e., equal to 1 . Thus the continuous $K$ is constant in $\mathrm{F}(n-1)$ and thus equal to 1.

Finally, to prove (3), let $T$ be a translation and set $\lambda=K(T)>0$. We have to show that $\lambda=1$; asserting the contrary, suppose with no loss of generality that $\lambda<1$. For any $p \in \partial \mathbf{H}_{\mathbb{K}}^{n}$, the sequence $T^{\nu}(p)$ tends to $\infty$ as $\nu \rightarrow+\infty$ in the $d_{\mathfrak{H}}$-topology. On the other hand,

$$
d\left(p, T^{\nu}(p)\right) \leq d(p, T(p))+\cdots+d\left(T^{\nu-1}(p), T^{\nu}(p)\right) \leq\left(1+\cdots+\lambda^{\nu-1}(p)\right) .
$$

But this is bounded in the $d$-topology and this contradicts (Top).

We finally prove that $K(\delta)=\delta^{\alpha}, \alpha \in(0,1]$. For this, observe that for every two arbitrary $\delta_{1}, \delta_{2}>0$ we have

$$
d\left(o, D_{\delta_{1}+\delta_{2}}(1,0)\right)=\left(\delta_{1}+\delta_{2}\right)^{\alpha} \cdot d(o,(1,0))
$$

and also by invariance of translations and triangle inequality,

$$
\begin{aligned}
d\left(o, D_{\delta_{1}+\delta_{2}}(1,0)\right) & =d\left(o,\left(\delta_{1}+\delta_{2}, 0\right)\right) \\
& =d\left(\left(-\delta_{1}, 0\right),\left(\delta_{2}, 0\right)\right) \\
& \leq \delta_{1}^{\alpha} \cdot d((-1,0), o)+\delta_{2}^{\alpha} \cdot d((1,0), o) \\
& =\delta_{1}^{\alpha} \cdot d((1,0), o)+\delta_{2}^{\alpha} \cdot d((1,0), o) \\
& =\left(\delta_{1}^{\alpha}+\delta_{2}^{\alpha}\right) \cdot d(o,(1,0)) .
\end{aligned}
$$

But also $d\left(o, D_{\delta_{1}+\delta_{2}}(1,0)\right)=\left(\delta_{1}+\delta_{2}\right)^{\alpha} \cdot d(o,(1,0))$; therefore, $\left(\delta_{1}+\delta_{2}\right)^{\alpha} \leq \delta_{1}^{\alpha}+\delta_{2}^{\alpha}$. By putting $\delta_{1}=\delta_{2}=\delta$ we have $2^{\alpha} \cdot \delta^{\alpha} \leq 2 \cdot \delta^{\alpha}$, i.e., $2^{1-\alpha} \geq 1$ which can happen only if $\alpha \in(0,1]$. This concludes the proof. 
Remark 3.2. (Notation convention) It is clear from the proof of Lemma 3.1 that

$$
d\left(o,\left( \pm \mathbf{e}_{i}, \mathbf{0}_{\Im(\mathbb{K})}\right)\right)=d\left(o,\left(\mathbf{e}_{1}, \mathbf{0}_{\Im(\mathbb{K})}\right)\right) .
$$

where $\mathbf{e}_{i}$ is the $i$-coordinate vector of $\mathbb{K}^{n-1}$. All these quantities shall be hereafter denoted by $d((1,0), o)$. Also

$$
d\left(o,\left(\mathbf{0}_{\mathbb{K}^{n-1}}, \pm \mathbf{f}_{i}\right)\right)=d\left(o,\left(\mathbf{0}_{\mathbb{K}^{n-1}}, \mathbf{f}_{1}\right)\right) .
$$

where $\mathbf{f}_{i}$ is the $i$-unit vector of $\Im(\mathbb{K})$. This is is true because according to Proposition $2.4, J$ is in $\operatorname{Sim}_{d}$; in particular, $K(J)=1$. All these quantities shall be denoted by $d((0,1), o)$.

3.2. Proof of Theorem 1.1. For the case $\mathbb{K}=\mathbb{R}$ (case (1) of the theorem), let $\zeta, \zeta^{\prime} \in \mathbb{R}^{n-1}$. We have

$$
\begin{aligned}
d\left(\zeta, \zeta^{\prime}\right) & =d\left(\zeta-\zeta^{\prime}, 0\right) \\
& =d\left(\left\|\zeta-\zeta^{\prime}\right\| \cdot \mu, 0\right), \quad \mu \in \mathrm{O}(n-1) \\
& =d(0,1) \cdot\left\|\zeta-\zeta^{\prime}\right\|^{\alpha}=d(0,1) \cdot d_{\mathfrak{H}}^{\alpha}\left(\zeta, \zeta^{\prime}\right)
\end{aligned}
$$

Here, the penultimate equation follows from the transitive action of $\mathrm{SO}(n-1)$ on $S^{n-1}$ : We may map any $r \in S^{n-1}$ to $1=\mathbf{e}_{1}$ via an element of $\operatorname{SO}(n-1)$.

For $\mathbb{K} \neq \mathbb{R}$ (case (2) of the theorem), let

$$
\beta_{1}=\max \{d((1,0), o), d((0,1), o)\}, \quad \beta_{2}=\min \{d((1,0), o), d((0,1), o)\} .
$$

Since for each $p=(\zeta, v) \in \mathfrak{H}_{\mathbb{K}}$ :

$$
\begin{aligned}
& d((\zeta, 0), o)=d((1,0), o) \cdot\|\zeta\|^{\alpha}, \\
& d((0, v), o)=d((0,1), o) \cdot|v|^{\alpha / 2},
\end{aligned}
$$

from 3.1 and 3.2 we obtain the inequalities:

$$
\begin{aligned}
& \beta_{2} \cdot\|\zeta\|^{\alpha} \leq d((\zeta, 0), o) \leq \beta_{1} \cdot\|\zeta\|^{\alpha} \\
& \beta_{2} \cdot|v|^{\alpha / 2} \leq d((0, v), o) \leq \beta_{1} \cdot|v|^{\alpha / 2}
\end{aligned}
$$

Therefore,

$$
\beta_{2} \cdot d_{\mathfrak{H}}^{\alpha}(o, p) \leq\left(d^{4 / \alpha}((\zeta, 0), o)+d^{4 / \alpha}((0, v), o)\right)^{\alpha / 4} \leq \beta_{1} \cdot d_{\mathfrak{H}}^{\alpha}(o, p) .
$$

By triangle inequality and $d$-invariance of translations we have

$$
d(p, o) \leq d((\zeta, 0), o)+d((0, v), o) .
$$

We apply Hölder's inequality with exponent $4 / \alpha$ to obtain

$$
\begin{aligned}
d(p, o) & \leq 2^{(4-\alpha) / 4} \cdot\left(d^{4 / \alpha}((\zeta, 0), o)+d^{4 / \alpha}((0, v), o)\right)^{\alpha / 4} \\
& \leq \beta_{1} \cdot 2^{(4-\alpha) / 4} \cdot\left(d_{\mathfrak{H}}^{4}((\zeta, 0), o)+d_{\mathfrak{H}}^{4}((0, v), o)\right)^{\alpha / 4} \\
& =\beta_{1} \cdot 2^{(4-\alpha) / 4} \cdot d_{\mathfrak{H}}^{\alpha}(p, o),
\end{aligned}
$$

and Theorem 1.1 follows.

We wish to address at one important issue at this point. As we have underlined in the introduction, condition $(\alpha-\mathbf{H o ̈ l})$ is rather inadequate to describe in full the nature of metrics which satisfy (Sim) and (Top); there might exist metrics which satisfy ( $\alpha$-Höl) on the one hand but on the other, their nature might be entirely different from that of $d_{\mathfrak{H}}$. A concrete example which illustrates this matter is that of the Carnot-Carathéodory metric $d_{c c}$ (for details about $d_{c c}$, see for instance [4]); it suffices only to consider the case $\mathbb{K}=\mathbb{C}, n=2$. Certainly, since $\operatorname{Sim}_{d_{c c}}=\operatorname{Sim}_{d_{\mathfrak{H}}}$, 
$d_{c c}$ automatically satisfies conditions (Sim) and (Top) of Theorem 1.1 (but satisfies neither (Inv) nor $(\mathbf{G}))$. Since $\alpha=1=\beta_{1}$, we have from Theorem 1.1 that for each $p$,

$$
d_{c c}(p, o) \leq 2^{3 / 4} \cdot d_{\mathfrak{H}}(p, 0)
$$

The reader is invited to compare this inequality to the well known estimate

$$
\pi^{-1 / 2} \cdot d_{\mathfrak{H}}(p, o) \leq d_{c c}(p, o) \leq \cdot d_{\mathfrak{H}}(p, o) .
$$

3.3. Proof of Theorem 1.2. The following lemma is an immediate corollary of Proposition 2.7; it will be needed for the proof of Theorem 1.2.

Lemma 3.3. If inversion I is in $\mathcal{M}_{d}\left(\mathfrak{H}_{\mathbb{K}}\right)$, then

$$
d(o, p) \cdot d(o, I(p))=d\left(o, p_{0}\right) \cdot d\left(o, I\left(p_{0}\right)\right),
$$

where $p_{0}$ is any point other than $o, \infty$ and for each $p \in \mathfrak{H}_{\mathbb{K}}$ other than $o, \infty$. Moreover

$$
d(I(p), I(q))=\frac{d(p, q)}{d(o, p) \cdot d(o, q)} \cdot d\left(o, p_{0}\right) \cdot d\left(o, I\left(p_{0}\right)\right),
$$

for each $p, q \in \mathfrak{H}_{\mathbb{K}}$ other than $o, \infty$.

We proceed now to the proof of Theorem 1.2. Recall that $J$ is in $\operatorname{Sim}_{d}$ and $K(J)=1$. By choosing $p_{0}=(1,0)$ which we shall denote simply with 1 , the formulae in the statement of the proposition also read as:

$$
d(o, p) \cdot d(o, I(p))=d^{2}(o, 1), \quad d(I(p), I(q))=\frac{d(p, q)}{d(o, p)} \cdot d(o, q) \cdot d^{2}(o, 1) .
$$

Let now $p=(\zeta, v) \in \mathfrak{H}_{\mathbb{K}}$. Then by setting $\mathcal{A}(p)=-\|\zeta\|^{2}+v$ we have

$$
\begin{aligned}
d(I(p), o)) & =d\left(\left(\zeta \cdot \frac{\mathcal{A}(\bar{p})}{|\mathcal{A}(p)|^{2}}, \frac{\bar{v}}{|\mathcal{A}(p)|^{2}}\right), o\right) \\
& =d\left(D_{|\mathcal{A}(p)|^{-1}}\left(\zeta \cdot \frac{\mathcal{A}(\bar{p})}{|\mathcal{A}(p)|}, \bar{v}\right), o\right) \\
& =|\mathcal{A}(p)|^{-\alpha} \cdot d\left(\left(\frac{\mathcal{A}(p)}{|\mathcal{A}(p)|} \cdot \bar{\zeta}, v\right), o\right) \\
& =|\mathcal{A}(p)|^{-\alpha} \cdot d((-\bar{\zeta}, v), o) \\
& =|\mathcal{A}(p)|^{-\alpha} \cdot d((-\zeta,-v), o) \\
& =|\mathcal{A}(p)|^{-\alpha} \cdot d\left(p^{-1}, o\right) \\
& =|\mathcal{A}(p)|^{-\alpha} \cdot d\left(T_{p}\left(p^{-1}\right), o\right) \\
& =|\mathcal{A}(p)|^{-\alpha} \cdot d(o, p) .
\end{aligned}
$$

This gives

$$
d(I(p), o))=\frac{d^{2}(o, 1)}{d(o, p)}=|\mathcal{A}(p)|^{-\alpha} \cdot d(o, p),
$$

and by Lemma 3.3 we conclude

$$
d^{2}(o, p)=d^{2}(o, 1) \cdot|\mathcal{A}(p)|^{\alpha}=d^{2}(o, 1) \cdot d_{\mathfrak{H}}^{2 \alpha}(o, p),
$$

which proves Theorem 1.2. 
Note that implicit in the proof lies the following formula for the inversion $I$ : For each $p$,

$$
I(p)=\left(D_{|\mathcal{A}(p)|^{-1}} \circ J \circ R_{-\frac{\mathcal{A}(p)}{|\mathcal{A}(p)|}} \circ J\right)\left(p^{-1}\right) .
$$

This formula only dictates the manner that inversion $I$ is defined; it cannot be regarded as formula which expresses $I$ as a compound of similarities, due to the presence of $p^{-1}$.

3.4. Proof of Theorem 1.3. Suppose now that (G) holds, that is,

$$
d(o, p)=\left(d^{4 / \alpha}((\zeta, 0), o)+d^{4 / \alpha}((0, v), o)\right)^{\alpha / 4} .
$$

Then $\beta_{2} \cdot d_{\mathfrak{H}}^{\alpha}(o, p) \leq d(o, p) \leq \beta_{1} \cdot d_{\mathfrak{H}}^{\alpha}(o, p)$. From $d$-invariance and $d_{\mathfrak{H}}$-invariance of translations we have:

$$
\beta_{2} \cdot d_{\mathfrak{H}}^{\alpha}(p, q) \leq d(p, q) \leq \beta_{1} \cdot d_{\mathfrak{H}}^{\alpha}(p, q)
$$

therefore $d$ and $d_{\mathfrak{H}}^{\alpha}$ are metrically equivalent. But there exists also a $\beta \geq 1$ depending on $\beta_{1}, \beta_{2}$ such that

$$
\frac{1}{\beta} \cdot d_{\mathfrak{H}}^{\alpha}(p, q) \leq d(p, q) \leq \beta \cdot d_{\mathfrak{H}}^{\alpha}(p, q) .
$$

If (Eq) holds, then $\beta_{1}=\beta_{2}=\beta$ and $d=\beta \cdot d_{\mathfrak{H}}^{\alpha}$. In this manner we have proved Theorem 1.3.

We remark here that condition (biLip) is equivalent to that the identity map from $\left(\partial \mathbf{H}_{\mathbb{K}}^{n}, d\right)$ to $\left(\partial \mathbf{H}_{\mathbb{K}}^{n}, d_{\mathfrak{H}}\right)$ is bi-Lipschitz.

Finally, we prove:

Proposition 3.4. Suppose that condition (Sim) holds. Then conditions $(\mathbf{G})$ is equivalent to the following:

(P-L) For $\alpha \in(0,1]$ and for each $p, q \in \mathfrak{H}$,

$$
\begin{array}{r}
|p * q|^{4 / \alpha}+\left|p^{-1} * q\right|^{4 / \alpha}+\left|p * q^{-1}\right|^{4 / \alpha}+\left|p^{-1} * q^{-1}\right|^{4 / \alpha} \\
2\left(\left|\Pi_{\mathbb{K}^{n-1}}(p * q)\right|^{4 / \alpha}+\left|\Pi_{\mathbb{K}^{n-1}}\left(p^{-1} * q\right)\right|^{4 / \alpha}\right) \\
+ \\
\left|\Pi_{\Im(\mathbb{K})}(p * q)\right|^{4 / \alpha}+\left|\Pi_{\Im(\mathbb{K})}\left(p^{-1} * q\right)\right|^{4 / \alpha}+\left|\Pi_{\Im(\mathbb{K})}\left(p * q^{-1}\right)\right|^{4 / \alpha}+\left|\Pi_{\Im(\mathbb{K})}\left(p^{-1} * q^{-1}\right)\right|^{4 / \alpha},
\end{array}
$$

where $\Pi_{\mathbb{K}^{n-1}}$ and $\Pi_{\Im(\mathbb{K})}$ are projections of $\mathfrak{H}$ to $\mathbb{K}^{n-1}$ and $\Im(\mathbb{K})$, respectively.

Proof. To show that (P-L) implies (G), just set $q=o$ and use the fact that $\left|p^{-1}\right|=|p|$, due to $d$-invariance of translations which follows from (Sim) (cf. Lemma 3.1):

$$
d\left(o, p^{-1}\right)=d\left(T_{p}(o), T_{p}\left(p^{-1}\right)\right)=d(o, p) .
$$

To show that $(\mathbf{G})$ implies (P-L), just apply $(\mathbf{G})$ for the points $p * q, p^{-1} * q, p * q^{-1}$ and $p^{-1} * q^{-1}$.

3.5. Proof of Theorem 1.4. Assuming condition ( $\alpha$-Met) holds, we have for each $p, q, r, s$ points in $\partial \mathbf{H}_{\mathbb{K}}^{n}$ that

$$
\begin{aligned}
d_{\mathfrak{H}}^{\alpha}(p, r) \cdot d_{\mathfrak{H}}^{\alpha}(q, s) & \leq\left(d_{\mathfrak{H}}(p, q) \cdot d_{\mathfrak{H}}(r, s)+d_{\mathfrak{H}}(p, s) \cdot d_{\mathfrak{H}}(r, q)\right)^{\alpha} \\
& \leq d_{\mathfrak{H}}^{\alpha}(p, q) \cdot d_{\mathfrak{H}}^{\alpha}(r, s)+d_{\mathfrak{H}}^{\alpha}(p, s) \cdot d_{\mathfrak{H}}^{\alpha}(r, q),
\end{aligned}
$$

where the first inequality follows from the Ptolemaean property of $d_{\mathfrak{H}}$ and the second inequality holds because $\alpha \leq 1$. Thus $d$ satisfies (Ptol). 
Assume now that ( $\alpha$-Met) and (Circ) hold. Pick any $p, q, r, s$ lying in the Ptolemaean circle $\sigma$ and suppose with no loss of generality that $p$ and $s$ separate $q$ and $r$. We may also normalise so that $q=o$ and $r=\infty$. Then

$$
d_{\mathfrak{H}}^{\alpha}(s, o)+d_{\mathfrak{H}}^{\alpha}(p, 0)=d_{\mathfrak{H}}^{\alpha}(p, s)
$$

Therefore,

$$
d_{\mathfrak{H}}(p, s)=\left(d_{\mathfrak{H}}^{\alpha}(s, o)+d_{\mathfrak{H}}^{\alpha}(p, 0)\right)^{1 / \alpha} \geq d_{\mathfrak{H}}(s, o)+d_{\mathfrak{H}}(p, 0),
$$

since $1 / \alpha \geq 1$. But this contradicts triangle inequality unless $\alpha=1$. Moreover, we have that then $\sigma$ is an $\mathbb{R}$-circle and the proof is complete.

\section{REFERENCES}

[1] D.J. Alcock; Reflection groups on the octave hyperbolic plane. J. of Algebra 213 (1998), 467-498.

[2] S. Buyalo \& V. Schroeder; Möbius structures and Ptolemy spaces: boundary at innity of complex hyperbolic spaces. arXiv:1012.1699v1 [math.MG]

[3] S.M. Buckley, K. Falk \& D.J. Wraith; Ptolemaic inequality and CAT(0) Glasgow Math. J. 51 (2009) 301314.

[4] L. Capogna \& D. Danielli \& S.D. Pauls \& J.T. Tyson; An introduction to the Heisenberg group and the sub-Riemannian isoperimetric problem. Progress in Mathematics, 259. Birkhuser Verlag, Basel, 2007.

[5] E. Falbel; Geometric structures associated to triangulations as fixed point sets of involutions. Topol. and its Appl. 154 (2007), no. 6, 1041-1052. Corrected version in www.math.jussieu.fr/ falbel

[6] E. Falbel \& I.D. Platis; The PU $(2,1)$-configuration space of four points in $S^{3}$ and the cross-ratio variety. Math. Ann. 340 (2008), no. 4, 935-962.

[7] W. Goldman; Complex hyperbolic geometry. Oxford Mathematical Monographs. Oxford Science Publications. The Clarendon Press, Oxford University Press, New York, 1999.

[8] A. Hatcher. Algebraic Topology. Cambridge University Press, 2002.

[9] A. Korányi \& H.M. Reimann; The complex cross ratio on the Heisenberg group. Enseign. Math. (2) 33 (1987), no. 3-4, 291-300.

[10] G.D. Mostow; Strong rigidity of locally symmetric spaces. Ann. Math. stud. 78, Princeton University Press, New Jersey, 1973.

[11] S. Markham \& J.R. Parker; Jørgensen's inequality for metric spaces with applications to the octonions. Adv. Geom. 7 no. 1., (2007), 19-38.

[12] I.D. Platis; Cross-ratios and the Ptolemaean inequality in boundaries of symmetric spaces of rank 1. Geometriae Dedicata, 169, 187-208, 2014.

[13] I.D. Platis; The Psp(2,1)-configuration space of four points in $S^{7}$. In preparation.

[14] I.J. Schoenberg; A remark on M.M. Day's characterization of inner-product spaces and a conjecture of L.M. Blumenthal. Proc. Amer. Math. Soc. 3 (1952), 961-964.

E-mail address: jplatis@math.uoc.gr, viktor.schroeder@math.uzh.ch

Department of Mathematics and Applied Mathematics, University of Crete, University Campus, GR 70013 Voutes, Heraklion Crete, Greece

Institut für Mathematik, Universität Zürich, Winterthurerstrasse 190, CH 8057 ZüRICH, SwitZerland 